\title{
COVID-19 and Chronic Noncommunicable Diseases
}

\author{
Juan Carlos Mirabal Requena ${ }^{1 *}$ and Belkis Alvarez Escobar ${ }^{2}$ \\ ${ }^{1}$ Master in Natural Medicine and Bioenergetics, Second Degree Specialist in Comprehensive General Medicine, First Degree \\ Specialist in Physical Medicine and Rehabilitation, Assistant Principal Professor, Assistant Researcher, Sancti Spíritus University \\ of Medical Sciences, Rector, Sancti Spíritus, Cuba
}

${ }^{2}$ Master in Satisfactory Longevity, Second Degree Specialist in Comprehensive General Medicine, Assistant Principal Professor, Added Researcher, Sancti Spíritus University of Medical Sciences, Methodological Department, Sancti Spíritus, Cuba

*Corresponding author: Juan Carlos Mirabal Requena, Master in Natural Medicine and Bioenergetics, Second Degree Specialist in Comprehensive General Medicine, First Degree Specialist in Physical Medicine and Rehabilitation, Assistant Principal Professor, Assistant Researcher, Sancti Spíritus University of Medical Sciences, Rector, Sancti Spíritus, Cuba

\begin{tabular}{|c|c|}
\hline ARTICLE INFO & ABSTRACT \\
\hline Received: 㓞June 16, 2021 & $\begin{array}{l}\text { Citation: Juan Carlos Mirabal Requena, Belkis Alvarez Escobar. COVID-19 and Chronic } \\
\text { Noncommunicable Diseases. Biomed J Sci \& Tech Res 36(4)-2021. BJSTR. MS.ID.005897. }\end{array}$ \\
\hline
\end{tabular}

\section{Commentary}

Chronic non-communicable diseases (NCDs) are long-term conditions that are not caused by an acute infection, evolve slowly and are not transmitted from person to person. These diseases can be found in all age groups and constitute a public health problem because they are a cause of morbidity, within the framework of the aging process of the population worldwide and due to an unhealthy way of life. They are the leading cause of death and disability in the world. These diseases result in long-term health consequences and often create a need for long-term care and treatment. Other important conditions are also considered noncommunicable diseases, including injuries and mental health disorders [1]. Many NCDs can be prevented by reducing common risk factors, such as tobacco use, harmful use of alcohol, physical inactivity, and eating unhealthy foods. Screening, monitoring, and treatment, as well as palliative care, are critical components of the response to NCDs. NCDs kill 41 million people each year, which is equivalent to $71 \%$ of the deaths that occur in the world. In the Region of the Americas, there are 5,5 million deaths from these diseases annually. Worldwide, an estimated 15 million people between the ages of 30 and 69 die from causes related to NCDs; more than $85 \%$ of these premature deaths occur in low- and middle-income countries. In the Region of the Americas, 2,2 million people die from NCDs before their 70th birthday [2].
Cardiovascular disease accounts for the majority of NCD deaths (17,9 million each year), followed by cancer (9,0 million), respiratory diseases (3,9 million), and diabetes (1,6 million), a world level. These four groups of diseases are responsible for more than $80 \%$ of all premature deaths [3]. In the current pandemic that affects the population of all regions, it has been seen that the presence of NCDs constitutes a poor prognosis for the evolution of the disease. NCDs act negatively on the immune system of people, which is violated by Microorganisms (bacteria, viruses, and fungi), Parasites, among others, this effect is seen with greater emphasis in people over 60 years of age. In this age group, the immune system loses the ability to detect foreign antigens, which is why autoimmune disorders are more frequent. It has been pointed out by researchers in different publications and information media on this subject, that the highest mortality occurs in patients over 60 years of age, as well as in carriers of NCDs [4]. Antibodies lose the ability to attack antigen. These changes partly explain why pneumonia, influenza, infective endocarditis, and tetanus are more common in older age and lead to death more often, vaccines are less effective in older age, and therefore it is important that people older booster doses are given (available for certain vaccines).

In the last 40 years, humanity has been affected by three major pandemics that have claimed many deaths: the increase in 
the incidence and prevalence rates of chronic non-communicable diseases (NCDs), the pandemic of the disease by the human immunodeficiency virus-AIDS (HIV / AIDS) and currently the pandemic caused by COVID-19. The latter, an emerging viral infectious disease with high mortality, which has contributed to an increase in mortality from NCDs in a very short time and has produced a collapse of health systems around the world [5]. NCDs take a higher level of concern among the scientific health guild for several reasons: the large number of affected cases with their well-known increasingly increasing incidence rates, their gradual contribution to general mortality, because they are the most frequent cause disability, due to the high cost of hospitalization, medical treatment and subsequent rehabilitation. Its emergence as a public health problem continues to be the result of several factors: social, cultural, political and economic changes that modified the style and living conditions of a large percentage of the population on a universal scale: the social determinants of health [6].

According to studies carried out, 163 countries reported that services for the care of NCDs had been totally or partially affected due to the health crisis. The inequality caused by the pandemic is being particularly echoed in the field of NCDs, so that COVID-19 and NCDs are presented as a syndemic, a pandemic made up of two diseases that exist simultaneously and have synergy with each other. which is interacting with and increasing social and economic inequalities [7]. The most recent scientific studies have shown that these diseases and their metabolic, behavioral and environmental risk factors are associated with a greater susceptibility to infection by SARS-CoV-2 and an increased risk of developing serious symptoms of COVID -19 and death. Alternatively, the pandemic has negatively affected the resources allocated to diagnosis, treatment rehabilitation and palliative care for these patients, at the same time that the confinement measures have led to an increase in behavioral risk factors for the development and evolution of the non-communicable diseases, since it is associated with more physical inactivity, poor diet and higher alcohol consumption. COVID-19 is interacting with NCDs and inequalities and has spawned the perfect storm of preventable death and suffering [8].

It is estimated that almost a quarter, $22 \%$, of the world's population suffers from a pre-existing disease that makes them

\section{ISSN: 2574-1241}

DOI: 10.26717/BJSTR.2021.36.005897

Juan Carlos Mirabal Requena. Biomed J Sci \& Tech Res

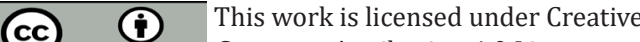

Commons Attribution 4.0 License

Submission Link: https://biomedres.us/submit-manuscript.php more vulnerable to COVID-19; most of these diseases are NCDs. For this reason, care at the Primary Health Level reaches such importance these days in the control and treatment of NCDs, but also in the investigation of possible positive or suspected cases of COVID-19 among the fragile or vulnerable population.

It is essential to inform the public about these health risks posed by COVID-19. In addition, health services must adapt to maintain essential services for NCDs to ensure continuity of care during the COVID-19 pandemic. This aspect is fundamental, since many times the greatest amount of resources, both human and material, is dedicated to the direct care of the patient with COVID and in a certain way the control and prevention of NCDs is neglected. Strengths in health are a fundamental pillar to deal with the current pandemic situation. But it must be accompanied by another important range of economic, social, mobility and telecommunications measures, among others, that encourage the population to follow preventive actions aimed at limiting the spread of the virus. To this must be added robust epidemiological and statistical surveillance systems that offer daily information in a clear, direct and accessible way to the population.

\section{References}

1. (2021) Pan American Health Organization. Noncommunicable diseases.

2. (2019) Ministry of Public Health of the Republic of Cuba. Noncommunicable diseases in Cuba, how to reduce the risks of suffering from them?

3. (2021) Pan American Health Organization. Annual Report of the Director of the Pan American Sanitary Bureau 2020. Saving lives and improving health and well-being.

4. Yang J, Zheng Y, Gou X, Pu K, Chen Z, et al. (2020) Prevalence of comorbidities in the Novel Wuhan Coronavirus (COVID-19) infection: a systematic review and meta-analysis. J Inter Infect Dis 94(10): 91-95.

5. Serra VMA (2020) Chronic noncommunicable diseases and the COVID-19 pandemic. Rev Finlay 10(2): 78-88.

6. Serra MA, Serra M, Viera M (2018) Chronic non-communicable diseases: current magnitude and future trends. Finlay 8(2).

7. (2020) Infocop. COVID-19 and non-communicable diseases, the "perfect storm" - WHO and UN report.

8. (2020) World Health Organization and United Nations Development Program. Address noncommunicable diseases during and after the COVID-19 pandemic.

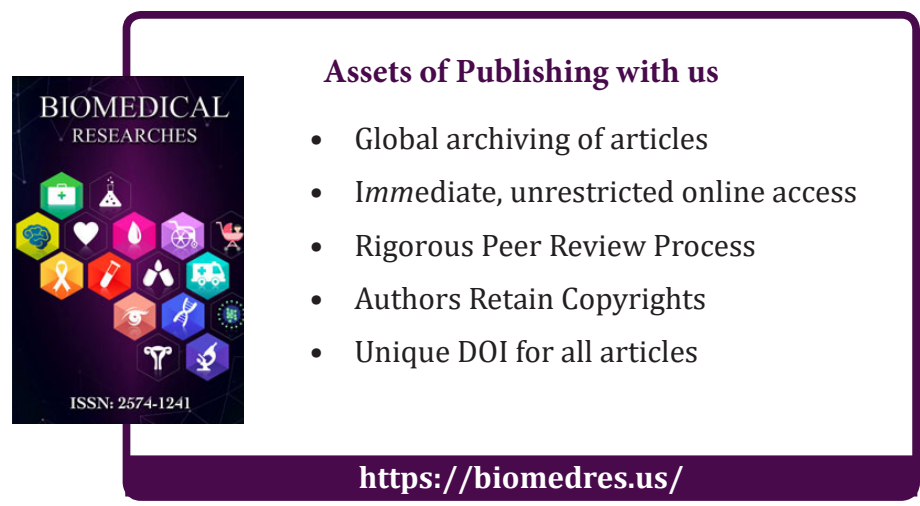

